\title{
Mantle Cell Lymphoma in the Orbital and Adnexal Region
}

Peter Rasmussen ${ }^{1}$, Lene D. Sjö ${ }^{1,2}$, Jan Ulrik Prause ${ }^{1}$, Elisabeth Ralfkiaer ${ }^{2}$ and Steffen Heegaard ${ }^{1}$

1. Department of Neuroscience and Pharmacology, Section of Eye Pathology, University of Copenhagen, Denmark

2. Department of Pathology, Copenhagen University Hospital, Denmark

CORRESPONDING AUTHOR:

Steffen Heegaard

Department of Neuroscience and Pharmacology, Section of Eye Pathology

Frederik V's Vej 11, 1

DK-2100 Copenhagen, Denmark

Phone: +45 35326070

Fax: +4535326080

E-mail: sh@eyepath.ku.dk

The Corresponding Author has the right to grant on behalf of all authors and does grant on behalf of all authors, an exclusive licence (or non exclusive for government employees) on a worldwide basis to the BMJ Publishing Group Ltd and its licensees, to permit this article (if accepted) to be published in BJO and any other BMJPG products and to exploit all subsidiary rights, as set out in our licence (http://bjo.bmjjournals.com/misc/ifora/licenceform.shtml)

Key words: Mantle cell - lymphoma - orbit - adnexa - treatment. 


\section{ABSTRACT}

Aims: To characterize clinicopathological features of mantle cell lymphoma (MCL) in the orbital and adnexal region.

Methods: Data of lymphoid lesions were retrieved searching the Danish Ocular Lymphoma Database 1980-2005. Specimens were collected from Danish pathological departments and reevaluated with a panel of monoclonal antibodies. For all patients with confirmed MCL the complete clinical files were collected and reviewed.

Results: Twenty-one patients with MCL in the orbital and adnexal region were identified comprising nine percent (21/230) of all lymphomas in the ocular region. There were 18 male- and three female patients with an age range from 60 to 90 years (median, 75 years). Orbital and adnexal region MCL as first presenting symptom comprised $67 \%$ of the patients. Of these, $71 \%$ had bilateral involvement. The orbit (71\%) and eyelids (64\%) were the most commonly affected sites. All but two presented in stage III/IV. Secondary MCL comprised 33\% of the patients. Bilateral affection (29\%) was less common in this patient group. Median survival was not different between the two presentation groups. Patients receiving anti-CD20 (rituximab)-containing chemotherapy had a significantly better five-year overall survival (OS) rate (83\%) than patients in treatment regimes without rituximab (five-year OS rate, $8 \%$ ).

Conclusions: Orbital and adnexal region MCL presents in elderly males. The orbit and eyelid are frequently involved. There is a very high proportion of systemic involvement in general with MCL of the orbital and adnexal region. Most patients presented with stage IV disease and had multiple relapses and short survival time. Treatment with rituximab-containing chemotherapy improved survival significantly compared to combination chemotherapy without rituximab. 


\section{INTRODUCTION}

Mantle cell lymphoma (MCL) is a rare type of malignant lymphoma representing 3-5\% of all nonHodgkin lymphomas.[1] In 1992 the International Lymphoma Study Group recognized MCL as an independent malignant lymphoma with origin in peripheral B-cells of the inner mantle zone, characterized by $\mathrm{t}(11 ; 14)(\mathrm{q} 13 ; \mathrm{q} 32)$ and up-regulation of $b c l-1$ (cyclin D-1).[2] MCL is a disease of the elderly, with a male predominance, usually presenting with widespread disease and a relatively aggressive clinical course.[1, 3, 4] Management of MCL has been limited to palliative therapy, mainly consisting of anthracycline-based chemotherapy.[5] Recently, novel strategies combining anthracyclin-containing treatment i.e. cyclophosphamide, vincristine, doxorubicin and prednisone (CHOP), anti-CD20 agent (rituximab) and autologous stem cell transplantation (ASCT) have shown improved response- and survival rates.[6]

Morphologically, MCL resembles low-grade lymphoma, but due to overexpression of cyclin D-1, MCL is identified with standard immunohistochemical methods.[2]

In the orbital and adnexal region MCL represents one to five percent of all lymphomas.[7, 8] Clinicopathological reports of MCL in this region have only been published in a few studies with a limited number of cases.[9, 10]

The aim of the present study was to correlate the clinicopathological features with prognosis in the so far largest series of unselected patients with MCL in the orbital and adnexal region. 


\section{MATERIALS AND METHODS}

The patients were identified through the Danish Ocular Lymphoma Database covering the years 1980-2005. Formalin fixed, paraffin embedded specimens of identified patients were retrieved from the archives of several Danish pathology departments and sections were examined by immunohistochemistry for: bcl-2, bcl-6, CD3, CD5, CD10, CD20, CD23, CD79 $\alpha$, cyclin D-1 and MIB-1 (Ki-67). The staining was performed in the Techmate 500 Immunostainer, using the DAKO Envision K5007 as a secondary antibody. The samples were examined by two independent pathologists and reviewed in consensus, to reclassify the specimens according to the World Health Organisation Classification of Tumours 2001.[2]

Confirmed cases of MCL had to fulfil the following criteria: (1) Appearance of the characteristic morphology for MCL in a haematoxylin-eosin staining, specifically the presence of small- to medium-sized lymphoid cells with scant cytoplasm, irregular nuclei, inconspicuous nucleoli, and absence of large transformed cells; (2) Overexpression of nuclear cyclin D-1.

Patients with orbital and adnexal region MCL as first presenting symptom comprised cases with primary MCL and cases with orbital and adnexal lymphoma with non-diagnosed preexiting extraorbital disease.

\section{Clinical data}

Clinical data recorded for each patient included year of diagnosis, gender, age, symptoms and clinical findings, evidence of systemic involvement according to Ann Arbor staging Classification,[2] presence of B-symptoms, paraclinical findings inclusive serum lactate dehydrogenase (LDH) level, performance status according to the Eastern Cooperative Oncology Group (ECOG), score according to the MCL International Prognostic Index,[11] type of treatment and response, recurrence-free period, time and localisation of relapse, duration of survival, stage of disease at last follow-up and time and cause of death. The MCL International Prognostic Index score is based on four prognostic factors: age, performance status, lactate dehydrogenase and leukocyte count. 
Treatment response was measured as complete response (CR), partial response (PR), stable disease (SD) and progressive disease (PD). Complete response was defined as disappearance of all evidence of disease with complete resolution of previous abnormal investigations. Partial response was defined as regression of at least $50 \%$ of all measurable disease and no new sites of disease. Relapsed- or progressive disease was defined as appearance of any new lesion or increase by over $50 \%$ of previously involved sites.[12] Failure to reach CR/PR or PD was considered stable disease. The study was approved by the local ethics committee (KF 01262201 ) and the Danish Data Protection Agency (j. no. 2005-41-5098).

\section{Statistical analysis}

Overall survival (OS) was defined as the interval between the date of diagnosis in the orbital and adnexal region or at other sites for secondary MCL and last follow-up/contact or death whichever occurred first. Progression free survival (PFS) time was calculated from the date of diagnosis of MCL in the orbital and adnexal region to the date of first progression after initial treatment or most recent follow-up. PFS and OS were plotted by use of the Kaplan Meier method. Differences in OS were analyzed by the log-rank test and considered significant if $\mathrm{p}<0.05$. 


\section{RESULTS}

\section{Clinical features and location of disease}

A total of 21 cases $(9 \%)$ of MCL in the orbital and adnexal region were identified out of 230 Danish patients with lymphoma in the ocular region covering 25 years. These included $18(86 \%)$ male- and three (14\%) female patients with an age range from 60 to 90 years (median 73.4 years). The cases were evenly distributed over the period of 25 years. Patients with orbital and adnexal region MCL as first presenting symptom comprised $67 \%(14 / 21)$ of the cases (table 1).

Table 1 Clinical and paraclinical characteristics of 21 patients with mantle cell lymphoma in the orbital and adnexal region

MCL as first
presenting symptom

\begin{tabular}{|c|c|c|}
\hline & No. of patients $(\%)$ & No. of patients $(\%)$ \\
\hline \multicolumn{3}{|l|}{ Gender } \\
\hline Male & $12(86)$ & $6(86)$ \\
\hline Female & $2(14)$ & 1 (14) \\
\hline \multicolumn{3}{|l|}{ Age at presentation in the OAR } \\
\hline$\leq 60$ years & $0 \quad(0)$ & $1(14)$ \\
\hline$>60$ years & $14(100)$ & $6 \quad(86)$ \\
\hline Primary MCL in the OAR & $1 \quad(7)$ & - \\
\hline MCL in the OAR with preexisting extraocular disease & $13(93)$ & $7(100)$ \\
\hline \multicolumn{3}{|l|}{ Laterality } \\
\hline Unilateral & $4 \quad(29)$ & $5 \quad(71)$ \\
\hline Bilateral & $10(71)$ & $2(29)$ \\
\hline \multicolumn{3}{|l|}{ Bone marrow involvement } \\
\hline Present & $11(79)$ & $4(57)$ \\
\hline Absent & $3(21)$ & $3(43)$ \\
\hline \multicolumn{3}{|l|}{ Nodal involvement at diagnosis in the OAR } \\
\hline Present & $13(93)$ & $5 \quad(71)$ \\
\hline Absent & $1 \quad(7)$ & $2(29)$ \\
\hline \multicolumn{3}{|l|}{ Serum LDH level } \\
\hline Normal & $8(57)$ & $5(71)$ \\
\hline Elevated & 4 (29) & $2(29)$ \\
\hline Not analyzed & $2(14)$ & $0 \quad(0)$ \\
\hline \multicolumn{3}{|l|}{ Haemoglobin level } \\
\hline Normal & $13(93)$ & $3(43)$ \\
\hline Below normal & $1(7)$ & $4(57)$ \\
\hline \multicolumn{3}{|l|}{ MCL International Prognostic Index } \\
\hline LR, prognostic score $<5.7$ & 11 (79) & $6(86)$ \\
\hline IR, prognostic score $\geq 5.7$ or $<6.2$ & $0 \quad(0)$ & $0 \quad(0)$ \\
\hline HR, prognostic score $\geq 6.2$ & $0 \quad(0)$ & $0 \quad(0)$ \\
\hline Not analyzed & $3(21)$ & 1 (14) \\
\hline \multicolumn{3}{|l|}{ Ann Arbor staging at presentation in the OAR* } \\
\hline Stage I & $1 \quad(7)$ & $0 \quad(0)$ \\
\hline Stage II & $1 \quad(7)$ & $0 \quad(0)$ \\
\hline Stage III & $1 \quad(7)$ & $0 \quad(0)$ \\
\hline Stage IV & $11 \quad(79)$ & $7(100)$ \\
\hline
\end{tabular}

Abbreviations: HR: High risk; IR: Intermediate risk; LDH: Lactate Dehydrogenase; LR: Low risk;

MCL: Mantle cell lymphoma; OAR: Orbital and adnexal region; *: Complete staging was done in only seven of the 14 patients $(50 \%)$ with MCL as first presenting symptom. The remaining seven patients had a less extensive work-up. However, all 14 patients had bone marrow examination. 
The most frequent symptoms in this patient group were irritation/ pain, epiphora and diplopia, whereas the most frequent clinical signs were eyelid mass, conjunctival swelling and proptosis (table 2).

Table 2 Frequency of symptoms and clinical signs at presentation of mantle cell lymphoma in the orbital and adnexal region

\section{MCL as first presenting symptom}

Secondary MCL

\begin{tabular}{lrr}
\multicolumn{1}{c}{ Presenting symptoms/signs } & $\mathbf{n}(\boldsymbol{\%})$ & $\mathbf{n}(\boldsymbol{\%})$ \\
\hline Symptoms* & & \\
Irritation/ pain & $8(57)$ & $2(29)$ \\
Epiphora & $7(50)$ & $1(14)$ \\
Diplopia & $5(36)$ & $0(0)$ \\
Swelling & $4(28)$ & $4(58)$ \\
Proptosis & $2(14)$ & $2(29)$ \\
Ptosis & $2(14)$ & $0(0)$ \\
Decreased visual accuracy & $2(14)$ & $0(0)$ \\
Clinical signs* & & \\
Eyelid mass & $13(64)$ & $4(57)$ \\
Conjunctival mass & $8(36)$ & $3(43)$ \\
Proptosis & $7(36)$ & $2(29)$ \\
Ptosis & $3(14)$ & $1(14)$ \\
Restricted eye movement & $3(14)$ & $1(14)$ \\
Lacrimal gland mass & $1(5)$ & $0(0)$ \\
\hline Abria
\end{tabular}

Abbreviations: MCL: Mantle cell lymphoma; * A total of more than 100\%, since patients may have more than one presenting symptom or sign.

Bilateral lymphoma was present in $71 \%(10 / 14)$ of the patients. All cases had involvement of more than one site in the orbital and adnexal region. The orbit $(71 \%)$ and eyelid $(64 \%)$ were the most frequently affected sites (table 3).

Table 3 Frequency of occurrence in orbital and adnexal region sites of mantle cell lymphoma

\section{MCL as first presenting symptom}

Secondary MCL

\begin{tabular}{lrr}
\multicolumn{1}{c}{ Location* } & $\mathbf{n}(\boldsymbol{\%})$ & $\mathbf{n}(\boldsymbol{\%})$ \\
\hline Orbit & $10(71)$ & $3(43)$ \\
Eyelids & $9(64)$ & $4(57)$ \\
Conjunctiva & $5(36)$ & $1(14)$ \\
Lacrimal gland & $1(7)$ & $1(14)$ \\
Lacrimal sac & $1(7)$ & $1(14)$ \\
\hline
\end{tabular}

Abbreviations: MCL: Mantle cell lymphoma; * A total of more than 100\%, since patients may have involvement of more than one orbital and adnexal region site.

A complete staging procedure at the time of diagnosis that included a full body positron emission tomography (PET) and/or computed tomography (CT) and/or magnetic resonance imaging (MRI) and bone marrow biopsy, was performed in half (7/14) of the patients. The remaining seven patients 
had a less extensive staging that included a chest X-ray, ultrasound of the orbit and/or CT of the orbit, sinuses and nasal cavity. All seven cases had bone marrow examination.

Two patients had non-stage III/IV disease at diagnosis. One patient had bilateral involvement of the orbit and conjunctiva (stage I) and one patient had disease involving the right lacrimal gland and regional lymph nodes (stage II). In both cases staging procedures were performed using PET to rule out systemic involvement. The patient in stage I achieved a complete response following radiotherapy, but the tumour spread to the brain four months later. The patient being in stage II had a complete response following CHOP but developed a relapse classified as stage IV disease 42 months after primary diagnosis. The remaining 12 patients were all classified as being in stage III/IV at diagnosis $(86 \%, 12 / 14)$.

Secondary MCL implicated 33\% (7/21) of the patients (table 1). These patients most frequently presented with swelling, irritation/ pain and proptosis. Frequent clinical signs comprised a mass of the eyelid and conjunctiva along with proptosis (table 2). Bilateral ocular disease was seen in $29 \%$ $(2 / 7)$ of these patients. Bone marrow involvement was observed in four patients $(57 \%)$ and of these three $(75 \%)$ had anaemia. All seven patients were classified as being in stage IV.

Eleven patients with orbital and adnexal region MCL as first presenting symptom and six patients with secondary MCL had sufficient clinical data to construct the MCL International Prognostic Index score. All the cases were located in the low risk group (score $<5,7$ ).

\section{Histologic and immunohistologic findings}

Diffuse growth pattern was demonstrated in $95 \%(20 / 21)$ of the cases, whereas one case $(1 / 21,5 \%)$ presented a nodular growth pattern (fig 1). The cellular marker for proliferation MIB-1 (Ki-67 labelling index) was assessed in all cases with a median of $13 \%$ (range, 5-40\%).

Immunohistochemically, all 21 specimens were positive for CD20, CD79 $\alpha$, BCL-2 and cyclin D-1, and negative for CD3, CD10, CD23 and bcl-6. All but five showed CD5 co-expression.

\section{Treatment}

Six of the 21 patients were given CHOP in combination with anti-CD20 treatment (rituximab). Two of these patients had additionally an autologous stem cell transplantation (ASCT). Another seven 
patients were treated with anthracyclin-containing regimes without rituximab and two patients received additional orbital radiotherapy. Six patients received an alkylating agent, chlorambucil or cyclophosphamide, in combination with prednisolone, and two of these were also treated with orbital radiotherapy. Two patients received only radiotherapy.

\section{Treatment outcome and survival data}

Twelve patients (86\%) with orbital and adnexal region MCL as first presenting symptom experienced a relapse or progression of disease with a median of 27.5 months during a follow-up period ranging from one to 62 months. Eight of the patients $(67 \%)$ had their relapse in the orbital and adnexal region, four (50\%) with bilateral involvement.

Five patients (71\%) with secondary lymphoma had a relapse or progression with involvement of the orbital and adnexal region in one case, orbital and adnexal region and systemic disease in three cases and systemic relapse in one case.

Estimated median PFS for all patients was 17 months from the time of diagnosis in the orbital and adnexal region. Median OS rate for patients with orbital and adnexal region MCL as first presenting symptom was 43 months and 51 months for patients with systemic presentation of MCL (fig 2). Patients with orbital and adnexal region mantle cell lymphoma as first presenting symptom had a significantly shorter maximum survival $(\mathrm{p}<0.04)$, than patients with systemic presentation of MCL. Patients treated with CHOP, rituximab and bone marrow transplantation were lumped with patients receiving only CHOP plus rituximab $(n=6)$, since the number of patients was too small to perform statistical analysis. This group had a five-year OS of $83 \%$, which was significantly higher $(\mathrm{p}<0.027)$ than the group of patients $(\mathrm{n}=13)$ receiving chemotherapeutic treatment without rituximab (five-year OS rate, 8\%) (fig 3). Among the 14 patients who died during follow-up (14/21, 67\%), 13 (93\%) died from lymphoma progression three to 62 months after diagnosis (median: 27 months). 


\section{DISCUSSION}

Mantle cell lymphoma is a non-Hodgkin lymphoma, described almost four decades ago as centrocytic lymphoma and recognized by the small cleaved cells with irregular nuclei, fine chromatin and small or absent nucleoli.[13] In 1992, MCL was recognized as an independent lymphoma subtype by the International Lymphoma Study Group.[14] The European Task Force on Lymphoma further defined MCL in 1994.[15] With the availability of fluorescence in situ hybridization (FISH) it became evident that the $t(11 ; 14)(\mathrm{q} 13 ; \mathrm{q} 32)$ causing cyclin D-1 overexpression was present in virtually all cases.[16] Clinically, MCL is a lymphoma with a male predominance with multiple lymph node involvement and also bone marrow involvement.[14]

In the orbital and adnexal region MCL has previously been described in reports primarily from centers in North America and Europe,[7, 17] but only two studies with a limited number of cases focused on orbital and adnexal region MCL with clinical data.[9, 10] In our study we have analyzed 21 patients with orbital and adnexal region MCL in a relatively stable population of 5.2 million people over a period of 25 years and correlated the clinicopathological features with prognosis.

The proportion of orbital and adnexal region MCL was nine percent of all orbital and adnexal region lymphomas in Denmark.[18] This is slightly higher than previously described in the literature, reporting approximately four percent.[7, 17] There was no change of incidence over the study period in contrast to an increasing incidence reported in all ocular region lymphomas.[18] However, our findings of a steady incidence are in accordance with large reports on systemic MCL.[1]

We found that patients with MCL in the orbital and adnexal region were above 60 years of age and with a noticeable male predominance (male/female ratio $=6$ ), that exceeds observations of systemic MCL (male/female ratio = 3.1).[4] Mantle cell lymphoma was primarily located in the orbit and eyelid and interestingly, bilateral disease was observed in about three fourths of the patients with primary MCL and in one third of the patients with secondary MCL. In other orbital and adnexal 
region lymphoma bilateral location is only seen in up to $10 \%$.[18] The high percentage of bilateral disease might be explained by the fact that MCL presents with widespread disease.

The majority of patients in this series presented in stage IV with nodal- and bone marrow involvement and corroborate that there is a very high proportion of systemic involvement in general with MCL of the orbital and adnexal region.[19] Relapse or progression involving both ocular- and extraocular sites was seen in $86 \%$ of the patients and for the most part within two years after the diagnosis of orbital and adnexal region MCL. Bone marrow examination confirmed that the majority of patients presented in stage IV. However, interpretation of treatment response and outcome in this series may be of limited value due to the fact that the standardized guidelines changed during the 25-year study period in line with the availability of new imaging technologies.[12]

Median OS was 43 months for patients with orbital and adnexal region MCL as first presenting symptom and 51 months for patients with secondary MCL. This confirms that MCL patients have a poor prognosis.[3] Remarkably, there was a significantly longer maximum survival among patients with secondary MCL as opposed to patients with primary MCL. Also primary MCL had bone marrow involvement in $79 \%$ as opposed to $57 \%$ in secondary MCL.

Recently, the MCL International Prognostic Index has been established to replace the previously used International Prognostic Index and Follicular International Prognostic Index developed for diffuse large cell- and follicular lymphoma patients.[11] When applied on the 17 patients in our material, with sufficient information to construct the score, all patients were allocated in the low risk group with a predicted five-year OS rate of $60 \%$. Thus, in our cohort of patients the MCL International Prognostic Index score was only reliable as a prognostic tool for the subgroup of secondary MCL patients with a relatively indolent course of disease.

MCL has previously been considered incurable and treatment has been palliative, mainly in the form of anthracycline- or alkylating antineoplastic agents.[20] Recently, new treatment strategies with $\mathrm{CHOP}$ in combination with the anti-CD20 agent, rituximab, have shown improved responseand survival rates in patients with newly diagnosed MCL.[6] In our study, it was only possible to 
compare survival data of patients receiving $\mathrm{CHOP}$ in combination with rituximab including bone marrow transplantation versus $\mathrm{CHOP}$ alone, since the number of patients was very limited. Therefore, it was not possible to determine the impact of bone marrow transplantation on survival. We recognize that it is possible that the improved survival in the group receiving rituximab may also be partly due to the fact that some of these patients had bone marrow transplantation as part of their treatment. Seven patients received chemotherapy without rituximab and responded with tumour regression. However, six patients were treated with rituximab-containing chemotherapy and two had also an ASCT (according to the 2nd Nordic MCL protocol) with a significantly better outcome compared to patients treated with chemotherapy without rituximab.

In conclusion, MCL in the orbital and adnexal region is rare and presents in elderly males. MCL is predominantly located in the orbit and eyelid. In our study bilateral involvement was frequently seen. Most patients presented with stage IV disease and had multiple relapses and short survival time. Treatment with rituximab-containing chemotherapy significantly improved survival. 


\section{ACKNOWLEDGEMENTS}

There are no competing interests in this article.

The study was supported by grants from The A.P. Møller Foundation for the Advancement of Medical Science, Øjenfonden and Øjenforeningen Værn om Synet. 


\section{REFERENCES}

1 Andersen NS, Jensen MK, de Nully Brown P, et al. A Danish population-based analysis of 105 mantle cell lymphoma patients: incidences, clinical features, response, survival and prognostic factors. Eur J Cancer 2002;38:401-8.

2 Jaffe E, Harris N, Stein H, et al. World Health Organisation Classification of Tumours. Pathology and Genetics of Tumours of Haematopoietic and Lymphoid Tissues. Lyon, France: IARC Press, 2001.

3 Samaha H, Dumontet C, Ketterer N, et al. Mantle cell lymphoma: a retrospective study of 121 cases. Leukemia 1998;12:1281-7.

4 Tiemann M, Schrader C, Klapper W, et al. Histopathology, cell proliferation indices and clinical outcome in 304 patients with mantle cell lymphoma (MCL): a clinicopathological study from the European MCL Network. Br J Haematol 2005;131:29-38.

5 Barista I, Romaguera JE, Cabanillas F. Mantle-cell lymphoma. Lancet Oncol 2001;2:141-8.

6 Schulz H, Bohlius J, Skoetz N, et al. Chemotherapy plus Rituximab versus chemotherapy alone for B-cell non-Hodgkin's lymphoma. Cochrane Database Syst Rev 2007:CD003805.

7 Coupland SE, Hellmich M, Auw-Haedrich C, et al. Prognostic value of cell-cycle markers in ocular adnexal lymphoma: an assessment of 230 cases. Graefes Arch Clin Exp Ophthalmol 2004;242:130-45.

8 Ferry JA, Fung CY, Zukerberg L, et al. Lymphoma of the ocular adnexa: A study of 353 cases. Am J Surg Pathol 2007;31:170-84.

9 Looi A, Gascoyne RD, Chhanabhai M, et al. Mantle cell lymphoma in the ocular adnexal region. Ophthalmology 2005;112:114-9.

10 Hon C, Chan RT, Ma ES, et al. Lymphomatous proptosis as a novel feature of mantle cell lymphoma. Leuk Lymphoma 2006;47:71-5.

11 Hoster E, Dreyling M, Klapper W, et al. A new prognostic index (MIPI) for patients with advanced-stage mantle cell lymphoma. Blood 2008;111:558-65.

12 Cheson BD, Pfistner B, Juweid ME, et al. Revised response criteria for malignant lymphoma. J Clin Oncol 2007;25:579-86.

13 Swerdlow SH, Williams ME. From centrocytic to mantle cell lymphoma: a clinicopathologic and molecular review of 3 decades. Hum Pathol 2002;33:7-20. 
14 Banks PM, Chan J, Cleary ML, et al. Mantle cell lymphoma. A proposal for unification of morphologic, immunologic, and molecular data. Am J Surg Pathol 1992;16:637-40.

15 Zucca E, Stein H, Coiffier B. European Lymphoma Task Force (ELTF): Report of the workshop on Mantle Cell Lymphoma (MCL). Ann Oncol 1994;5:507-11.

16 Vaandrager JW, Schuuring E, Zwikstra E, et al. Direct visualization of dispersed 11q13 chromosomal translocations in mantle cell lymphoma by multicolor DNA fiber fluorescence in situ hybridization. Blood 1996;88:1177-82.

17 Fung CY, Tarbell NJ, Lucarelli MJ, et al. Ocular adnexal lymphoma: clinical behavior of distinct World Health Organization classification subtypes. Int J Radiat Oncol Biol Phys 2003;57:1382-91.

18 Sjo LD, Ralfkiaer E, Prause JU, et al. Increasing Incidence of Ophthalmic Lymphoma in Denmark from 1980 to 2005. Invest Ophthalmol Vis Sci 2008.

19 Hatef E, Roberts D, McLaughlin P, et al. Prevalence and nature of systemic involvement and stage at initial examination in patients with orbital and ocular adnexal lymphoma. Arch Ophthalmol 2007;125:1663-7.

20 Witzig TE. Current treatment approaches for mantle-cell lymphoma. J Clin Oncol 2005;23:6409-14. 


\section{LEGENDS}

\section{Figure 1}

A: $\quad$ Salmon-coloured tumour mass of the superior palpebral- and bulbar conjunctiva in a 67year-old male with mantle cell lymphoma.

B: $\quad$ Magnetic resonance imaging showing bilateral orbital tumour masses (asterisk) of the patient in figure $1 \mathrm{~A}$, demonstrating proptosis of the right eye. The tumours regressed completely in the orbital and adnexal region following radiotherapy but spread to the brain four months after primary diagnosis.

C: $\quad$ Mantle cell lymphoma showing a monotonous pattern of small cells. The nuclei are irregular with inconspicuous nucleoli and scant cytoplasm (Hematoxylin-eosin; x400).

D: $\quad$ Mantle cell lymphoma showing positive staining for cyclin D-1.

The majority of the neoplastic cells show a moderate to strong nuclear staining and only a faint cytoplasmic reaction $(\mathrm{x} 400)$.

\section{Figure 2}

Overall survival of mantle cell lymhoma in the orbital and adnexal region.

Median survival is not different between the two patient groups, however, mantle cell lymphoma in the orbital and adnexal region as first presenting symptom had a significantly shorter maximum survival.

': Censored patients; OAR: Orbital and adnexal region

\section{Figure 3}

Effect of anti-CD20 therapy on survival of mantle cell lymphoma in the orbital and adnexal region.

Patients receiving chemotherapy combined with anti-CD20 agents and bone marrow transplantation had a significantly better survival than patients receiving chemotherapy without anti-CD20 agents.

I: Censored patients; OAR: Orbital and adnexal region 


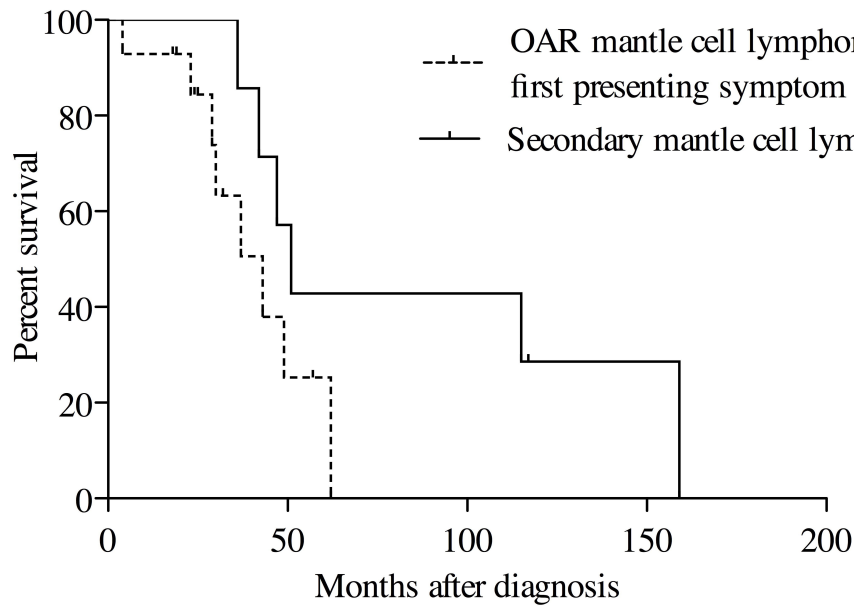

Figure 2 


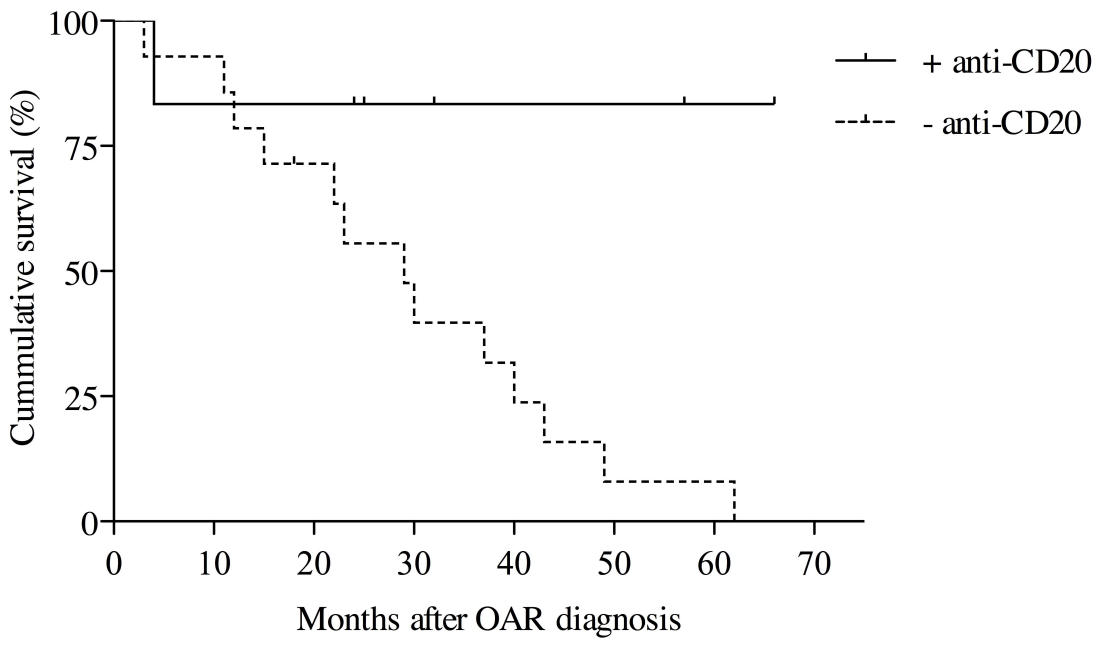

Figure 3 